\title{
Jugular phlebectasia presenting as globus pharyngeus
}

\author{
J. Czyżowski ${ }^{1}$, K.A. Tomaszewski ${ }^{2}$, J.A. Walocha ${ }^{2}$ \\ ${ }^{1}$ Department of Radiology, J. Dietl's Specialist Hospital, Krakow, Poland \\ 2Department of Anatomy, Jagiellonian University Medical College, Krakow, Poland
}

[Received 15 January 2013; Accepted 25 February 2013]

\begin{abstract}
Even though much has been written on the aetiology of globus pharyngeus, it still remains elusive and multifactorial. We present a case of a 54-year-old woman who was referred by an orthopedist to the radiology department with a 6-day history of an intense feeling of "pressure" and "tightness" in the jugular notch. After performing a contrast-enhanced computed tomography scan, a phlebectasia of the right anterior jugular vein was discovered. When trying to determine the cause of the globus sensation one has to consider the possible existence of a phlebectasia of one of the jugular veins. (Folia Morphol 2013; 72, 3: 278-280)
\end{abstract}

Key words: jugular phlebectasia, jugular vein, contrast-enhanced computed tomography, globus pharyngeus

\section{INTRODUCTION}

The first records of globus pharyngeus were written by Hippocrates 2500 years ago [3]. In 1707 Purcell described the condition as "globus hystericus" - the word "globus" originating from Latin, meaning "ball" and "hystericus" reflecting the assumed psychological component of the disorder [1]. Purcell believed that globus resulted from the pressure on the thyroid cartilage due to contraction of the strap muscles of the neck [3]. In 1968 Malcolmson [6] created the term "globus pharyngeus", after discovering that most of the patients experiencing globus have not had a hysterical personality.

Even though much has been written on the etiology of globus, it still remains poorly understood. It is most probably multifactorial, including gastroesophageal reflux disease, oesophageal motor disorders, pharyngeal inflammatory causes (such as pharyngitis, tonsillitis and chronic sinusitis), thyroid diseases, psychological factors and stress [1, 3]. Although data are limited, recent studies have focused on possible orga- nic causes. Due to the variety of potential aetiological factors it is difficult to establish a standard investigation and treatment strategy for the affected patients.

Phlebectasia of the jugular system is a congenital dilatation of the internal, external, anterior jugular vein or posterior facial vein. It is a rare, benign condition, most often asymptomatic [9]. It is usually encountered in older children and young adults [9]. More often it is unilateral than bilateral. Internal jugular phlebectasia is the most common form, while external, anterior jugular and posterior facial phlebectasias are uncommon [9]. Only 2 cases of right anterior jugular vein phlebectasia have been reported $[7,10]$ and non presenting as a globus pharyngeus.

In this paper we have described the first case of the right jugular vein phlebectasia presenting as a globus pharyngeus.

\section{CASE HISTORY}

A 54-year-old Caucasian woman with obesity and heart failure (NYHA II) was referred by an orthopedist

Address for correspondence: K.A. Tomaszewski, MD, Department of Anatomy, Jagiellonian University Medical College, ul. Kopernika 12, 31-034 Kraków, Poland, tel/fax: +48 1242295 11, e-mail: krtomaszewski@gmail.com 
to the radiology department with a 6-day history of an intense feeling of "pressure" and "tightness" in the jugular notch. A tumour of the sternoclavicular joint area was suspected.

She recalled having similar episodes over the last 30 years, but these became more intensive around the time she was diagnosed with heart failure. She also reported that the "pressure" and "tightness" around her throat increased whenever she came under stress. Currently, for the past 2 weeks, she was off her medications (ACE-inhibitor and diuretic) as she forgot to buy them. She did not smoke or drink alcohol and her family history was unremarkable.

Physical examination revealed a soft, reducible mass in the jugular notch. However, the Valsalva manouvre was negative. Laboratory tests and chest X-ray were unremarkable. The on-call radiologist decided to perform a contrast-enhanced computed tomography (CT) scan (Toshiba Aquilion CXL, 64-row detector) to rule out any pathologies that would not be detectable in a routine physical examination. The arterial phase did not reveal any abnormalities, however after assessing the venous phase scans, an abnormal, distended right anterior jugular vein, passing near the trachea (Figs. 1, 2), was discovered. The diameter of the right anterior jugular vein phlebectasia was approximately $11 \times 12 \mathrm{~mm}$.

The patient was evidently satisfied with the CT scan finding, as she remarked "now that I know what it is, the pressure 'on my throat' seems to have decreased". As the patient did not agree to surgical treatment, she was referred back to her primary care physician with a strong recommendation to regularly take her prescribed medications, avoid stress, control her diet and lose weight.

\section{DISCUSSION}

Globus pharyngeus has more recently been defined as (I) a persistent or intermittent sensation of a lump or foreign body in the throat for at least 12 weeks, (II) occurrence of the sensation between meals, (III) absence of dysphagia and odynophagia, (IV) absence of pathological gastroesophageal reflux, achalasia, or other motility disorder with a recognised pathological basis (e.g., scleroderma of the oesophagus) [2]. Our patient fully complies with the above-mentioned criteria and thus can be diagnosed with a globus pharyngeus. Though none of the presented etiological factors seem to fit the presented case.

On the basis of this case report we propose that venous abnormalities of the neck, like phlebectasia,

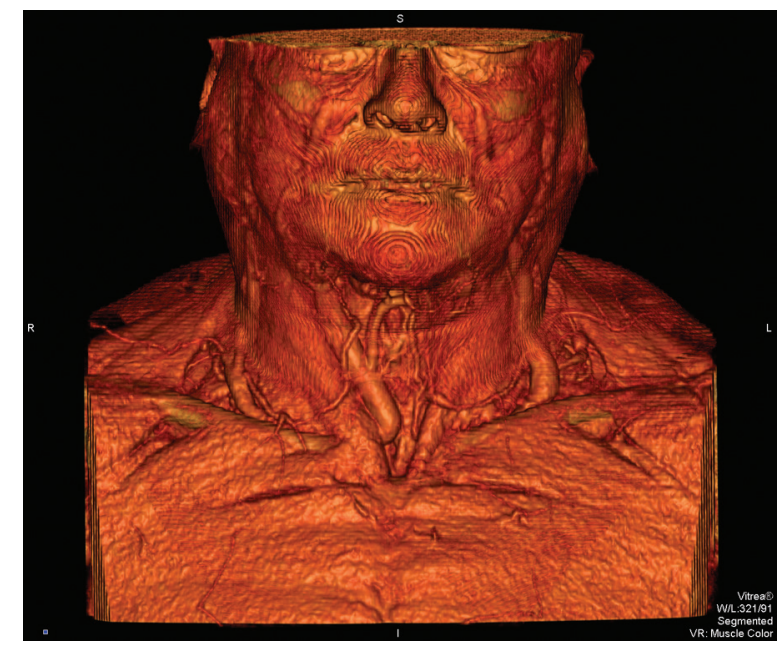

Figure 1. Virtual reconstruction of a contrast-enhanced computed tomography scan (venous phase). The right anterior jugular vein can be seen running under the sternocleidomastoideus muscle.

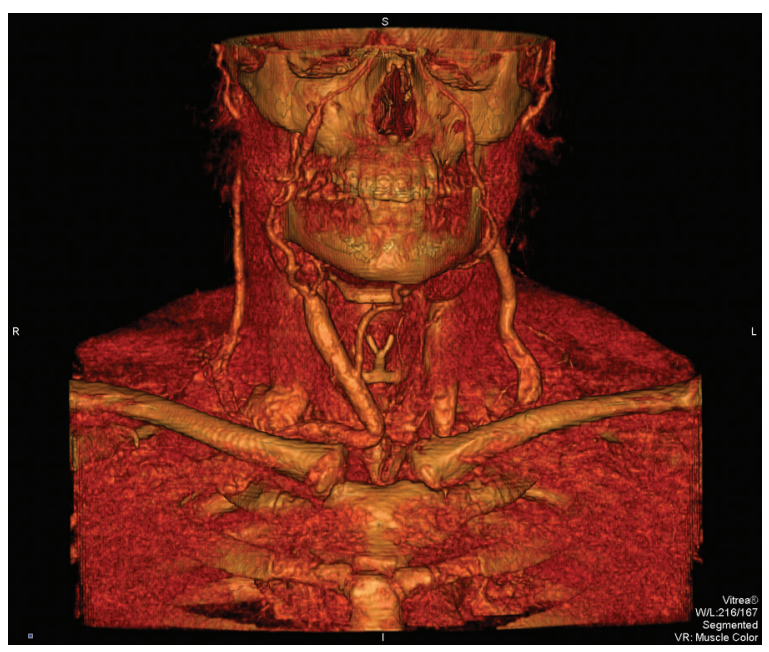

Figure 2. Virtual reconstruction of a contrast-enhanced computed tomography scan (venous phase). One can see that both sternoclavicular joints are free of any pathologies.

be considered a potential etiological factor of globus pharyngeus. A question arises why such a congenital malformation did not produce earlier globus sensations? The most probable answer lies in our patients' heart failure. The abnormal right anterior jugular vein started to press on the trachea only when, as a result of increased fluid intake and reduced heart function, hypervolaemia occurred. This increased the veins' diameter and allowed it to press on the trachea.

The aetiology of jugular phlebectasia remains unknown due to the rarity of such a finding. However, it is thought that congenital structural defects in the vein 
wall play an important role [5]. The majority of the cases are idiopathic [9]. Other possible causes include gross anatomic abnormality of the vein, mechanical compression or trauma of the vein and congenital structural defects in the vein wall [5]. Usually jugular phlebectasia presents as an asymptomatic, unilateral, soft, non-pulsative, painless and compressible neck mass, most noticeable during any type of straining or Valsalva manoeuvre, that disappears at rest $[9,10]$. The unusual element of this case, is the fact that phlebectasia usually presents itself in childhood, and is very rare in older patients.

Diagnosis of phlebectasia can usually be made on a clinical basis and confirmed by Doppler ultrasound and/or contrast-enhanced CT [5, 9]. The risk of a thrombus developing inside such a distended vein should not be higher than normal, as histopathology findings usually present a normal vein wall [5]. Treatment should only be initiated when phlebectasia becomes symptomatic or causes a cosmetic defect. Asymptomatic cases should undergo regular follow-up [5] and only be treated if the size of the phlebectasia regularly increases.

Our patient also associated her globus sensation with mental stress. On the basis of this case report it is difficult to find a link between these two. However, several studies have reported increased numbers of stressful life events preceding symptom onset, suggesting that life stress might be a co-factor in symptom genesis and in exacerbation. Up to $96 \%$ of patients with globus report symptom exacerbation during periods of high emotional intensity $[4,8]$.

\section{CONCLUSIONS}

In conclusion, when trying to determine the cause of a globus sensation one has to consider the possible existence of a phlebectasia of one of the jugular veins. A contrast-enhanced CT scan of the neck, with detailed venous phase assessment, should be performed to confirm or rule out such anatomical variations.

\section{REFERENCES}

1. Cashman EC, Donnelly MJ (2010) The natural history of globus pharyngeus. Int J Otolaryngol, DOI:10.1155/2010/159630.

2. Galmiche JP, Clouse RE, Bálint A, Cook IJ, Kahrilas PJ, Paterson WG, Smout AJ (2006) Functional esophageal disorders. Gastroenterology, 130: 1459-1465.

3. Harar RP, Kumar S, Saeed MA, Gatland DJ (2004) Management of globus pharyngeus: review of 699 cases. J Laryngol Otol, 118: 522-527.

4. Harris MB, Deary IJ, Wilson JA (1996) Life events and difficulties in relation to the onset of globus pharyngis. J Psychosom Res, 40: 603-615.

5. Hu X, Li J, Hu T, Jiang X (2005) Congenital jugular vein phlebectasia. Am J Otolaryngol, 26: 172-174.

6. Malcomson KG (1968) Globus hystericus vel pharyngis (a recommaissance of proximal vagal modalities). J Laryngol Otol, 82: 219-230.

7. Natarajan B, Johnstone A, Sheikh S, Palmer O, Madhavan KN (1994) Unilateral anterior jugular phlebectasia. J Laryngol Otol, 108: 352-353.

8. Thompson WG, Heaton KW (1982) Heartburn and globus in apparently healthy people. Can Med Assoc J, 126: 46-48.

9. Uzun C, Taskinalp O, Koten M, Adali MK, Karasalihoglu AR, Pekindil G (1999) Phlebectasia of left anterior jugular vein. J Laryngol Otol, 113: 858-860.

10. Zohar Y, Ben-Tovim R, Talmi YP (1989) Phlebectasia of the jugular system. J Craniomaxillofac Surg, 17: 96-98. 